\title{
PHOTOFIELD EMISSION SPECTROSCOPY OF Ba ON W
}

\author{
T. Radoń AND S. JaSKólKa
}

Institute of Experimental Physics, University of Wroclaw

Pl. Maxa Borna 9, 50-204 Wrocław, Poland

(Received September 19, 1994; in final form January 20, 1995)

Optical transitions in photofield emission from barium covered tungsten were observed. The initial energies of the excited electrons obtained for different crystallographic planes range near the values of $s_{1}=-0.07 \pm 0.02 \mathrm{eV}$ and $s_{2}=-0.28 \pm 0.04 \mathrm{eV}$ with respect to the Fermi level. The energies are ascribed to electrons in the thin barium layer. The work functions of the barium covered tungsten (100), (111) and (100) planes were estimated using field emission method. In order to measure very small photocurrents a modulated laser radiation and the phase-sensitive detection were used.

PACS numbers: $73.20 .-r, 79.70 .+q$

\section{Introduction}

It is well known that the adsorption of alkaline earth metals on transition metals reduces the work function of the substrates and this property is widely utilized in technology. The problem is to understand the surface electronic properties of the system such as Ba on W. Many years ago electron emission of this system was investigated by using the field emission method [1, 2]. In more recent years new experimental methods such as photoemission [3] and inverse photoemission [4] have been used. The Ba $5 d$ empty states of the atom are occupied in bulk barium. For the barium monolayer on aluminium no filled $d$ states are found [3] in contrast to the results obtained for $\mathrm{Ba}$ on $\mathrm{W}[2]$.

It seems to be useful to examine the $\mathrm{Ba} / \mathrm{W}$ system using photofield emission spectroscopy $[5,6]$. The method has proved to be very suitable to explore electronic states near the Fermi level, and detailed information for tungsten about its bulk band structure and the surface density of states $[7,8]$ was obtained. In this paper we present two optical excitations from thin barium films deposited on tungsten, which were obtained in the photofield emission characteristics.

\section{Experimental}

Measurements were performed using a field emission tube sealed off from a pumping system, a pressure of $10^{-9} \mathrm{~Pa}\left(10^{-11} \mathrm{Tr}\right)$ was maintained by a molybdenum getter [9]. The barium source was prepared from a small piece of barium 
evaporation getter (barium placed in a small iron boat) type used once in vacuum lamps. Barium was evaporated on the clean tungsten emitter tip from one side and then distributed over the surface by heating the emitter at a temperature of about $800 \mathrm{~K}$.

The tip was kept at room temperature during the measurements. The thin barium layer remained quietly on the tip surface at room temperature and did not produce a distinct noise in the photocurrent. To measure the very small photofield emission current component in the field emission current a modulation method [5] was employed. The laser light beam was chopped with the frequency of $8500 \mathrm{~Hz}$ and the alternating photocurrent was amplified using phase-sensitive detection. The angle of incidence of the light beam onto a plane of the tungsten crystal was between $65^{\circ}$ and $85^{\circ}$. The irradiance at the emitter tip was about $100 \mathrm{~W} / \mathrm{cm}^{2}$.

The lowering of the surface barrier was calculated from the geometrical factor $\beta$ of the emitter tip and the applied voltage. The factor $\beta$ was obtained from the Fowler-Nordheim characteristic assuming the work function to be $4.52 \mathrm{eV}$ for clean tungsten. The work functions of different barium-covered crystallographic planes were estimated by measuring field and photoemission charcteristics from the bulk at $s$-polarized light. The initial photoemission occurs from the Fermi level. In this case the work function is the sum of the quantum energy and the magnitude of the lowering of the surface barrier.

\section{Results}

Figure 1 shows photofield emission (PFE) characteristics obtained from the (110) plane of barium covered tungsten which was irradiated with $h \nu=1.96 \mathrm{eV}$. Curve $a$ was measured when the electric vector of the polarized light was parallel

TABLE

Optical excitations from the barium layer adsorbed on different planes of tungsten. Initial energies are calculated in relation to the Fermi level.

\begin{tabular}{c|c|c|c|c|c}
\hline \hline $\begin{array}{c}\text { Figure/ } \\
\text { /Curve }\end{array}$ & Plane & $\begin{array}{c}\text { Work } \\
\text { function } \\
\mathrm{Ba} / \mathrm{W}[\mathrm{eV}]\end{array}$ & $\begin{array}{c}\text { Excitation } \\
\text { energy [eV] }\end{array}$ & $\begin{array}{c}\text { Initial } \\
\text { energy } \\
{[\mathrm{eV}]}\end{array}$ & $\begin{array}{c}\text { Average } \\
\text { initial } \\
\text { energy [eV] }\end{array}$ \\
\hline $1 / b$ & $(110)$ & 4.11 & 1.96 & -0.05 & \\
- & $(110)$ & 4.66 & 2.54 & -0.09 & \\
- & $(110)$ & 3.99 & 1.96 & -0.09 & $S_{1}$ \\
- & $(111)$ & 3.37 & 1.96 & -0.05 & $-0.07 \pm 0.02$ \\
$2 / b$ & $(100)$ & 3.42 & 1.96 & -0.08 & \\
\hline $1 / b$ & $(110)$ & 4.11 & 1.96 & -0.23 & \\
- & $(110)$ & 4.66 & 2.54 & -0.32 & \\
- & $(110)$ & 3.99 & 1.96 & -0.27 & $S_{2}$ \\
- & $(111)$ & 3.37 & 1.96 & -0.30 & $-0.28 \pm 0.04$ \\
$2 / b$ & $(100)$ & 3.42 & 1.96 & -0.27 &
\end{tabular}




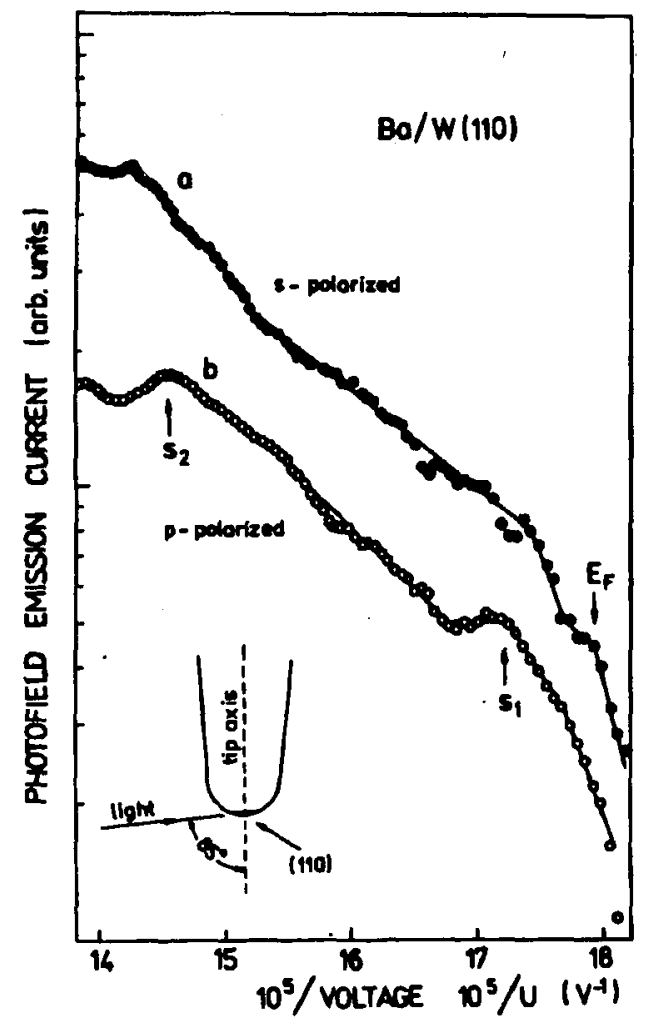

Fig. 1. Logarithmic plots of PFE current from the (110) plane of barium covered tungsten versus reciprocal voltage at the quantum energy $h \nu=1.96 \mathrm{eV}$. Curve $a$ was obtained using $s$-polarized light and curve $b$ using $p$-polarized light. The letter $E_{\mathrm{F}}$ indicates the transition from the Fermi level and letters $s_{1}$ and $s_{2}$ indicate the excitations from the surface layer. The direction of the lighting of the emitter is also shown in the figure.

to the emitting surface (s-polarized light) whereas curve $b$ was obtained for the perpendicular component of the electric vector ( $p$-polarized light). For the angle of incidence of the light beam equal to about $85^{\circ}$ the component perpendicular to the surface was prevailing. The estimated initial energies of electrons are given in Table.

Figure 2 contains PFE characteristics for the (100) plane of barium covered tungsten at $h \nu=1.96 \mathrm{eV}$. The angle of incidence of the light beam was equal to about $65^{\circ}$ when the perpendicular component of the electric vector was prevailing. The initial energies of electrons are also placed in Table.

The distinct shoulders $s_{1}$ and $s_{2}$ in Fig. 1 (curve $b$ ) are also visible in curve $a$ in which excitations from bulk transition should be prevailing. On the other hand, shoulder $E_{\mathrm{F}}$ in curve $a$ is also noticeable in curve $b$. This mixing of the excitations from the bulk and from the surface can be caused by a deflection of the light polarization in relation to the emitting-surface normal. Anyhow, the excitations in 


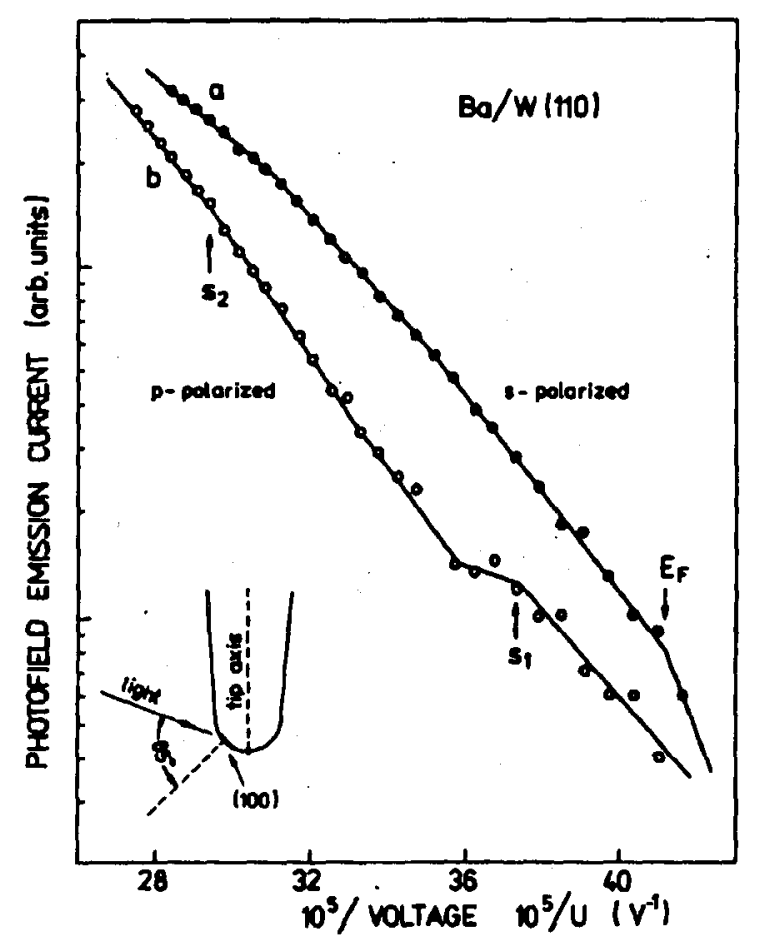

Fig. 2. Logarithmic plots of PFE current versus reciprocal voltage from the (100) plane of barium-covered tungsten at the quantum energy $h \nu=1.96 \mathrm{eV}$. Curve $a$ was obtained using $s$-polarized light and curve $b$ with $p$-polarized light. The letters $E_{\mathrm{F}}$ and $s_{1}, s_{2}$ indicate excitations from the Fermi level and the surface layer, respectively. The direction of the lighting of the emitter is also shown in the figure.

one curve are confirmed in the second one with a very small error. Similar remarks concern curves shown in Fig. 2, but the excitation $s_{1}$ from the surface is not visible in curve $a$. Curves in Fig. 1 were measured precisely using a computer program while curves in Fig. 2 were measured without computer.

\section{Discussion}

As it was earlier shown $[6,10]$ a shoulder in the photocurrent curve occurs at a voltage that lowers the top of the potential barrier at the emitter surface to the energy level of the excited electrons. Thus, the reduced barrier corresponds to the final energy of electrons above the Fermi level. Electrons can leave the Fermi level and pass at the level of the top of the barrier, which is manifested by the appearance of a shoulder in the curve at the lowest voltage. In Figs. 1 and 2 this shoulder is marked by $E_{\mathrm{F}}$. The energy of those electrons is equal to the quantum energy $h \nu$ used for illumination. In this situation the lowering of the barrier plus the distance of the top from the Fermi level is equal to the work function of a barium covered plane of tungsten. The lowering of the top of the barrier was 
calculated from the geometrical factor $\beta$ of the emitter and the voltage applied to the emitter [6].

While excitations appear in the bulk crystal (from the Fermi level) due to the $s$-polarized light, excitations in the surface prevail for the $p$-polarized light. It can be seen in curve $b$ of Fig. 1 , where the excitations $s_{1}$ and $s_{2}$ are more exhibited than in curve $a$, and excitation $E_{\mathrm{F}}$ from bulk tungsten is more exhibited in curve $a$ than in curve $b$. Since the same values of $S_{1}$ and $S_{2}$ were obtained for different barium covered tungsten planes, using different quantum energies, it suggests that the two energies should be ascribed to electrons of the barium layer.

All the results were obtained for thin barium films, less than a monolayer. The lowest work function obtained in this experiment was about $3.4 \mathrm{eV}$, whereas the work function of a barium monolayer on tungsten reaches the value of about $2 \mathrm{eV}$ [1]. This means that part of the tungsten planes were not adsorbate covered and the emission from $W$ surface states might be observed. But the energies of surface states theoretically calculated [11] and experimentally confirmed for clean (100) and (111) planes of tungsten [7,12] differ considerably from those observed for barium films (Fig. 3). These results confirm the conclusion mentioned that the excitations from different barium covered planes of tungsten concern electrons
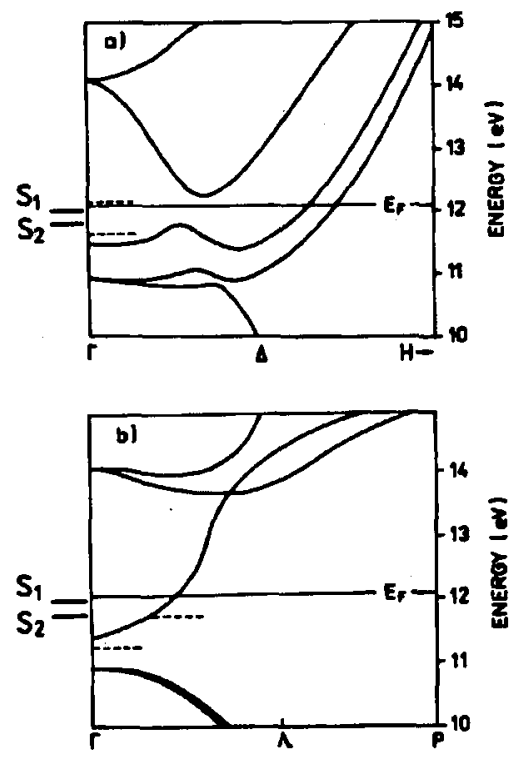

Fig. 3. The bulk band structure of tungsten near the Fermi level $E_{\mathrm{F}}$ : (a) Band structure of the tungsten $\langle 100\rangle$ direction, dashed lines parallel to the $E_{\mathrm{F}}$ indicate energies of surface states measured for clean tungsten (100) plane [7], $S_{1}$ and $S_{2}$ indicate energies measured for the barium covered plane. (b) Band structure of the tungsten $\langle 111\rangle$ direction, dashed lines parallel to the $E_{\mathrm{F}}$ indicate energies of surface states measured for clean tungsten (111) plane [12], $S_{1}$ and $S_{2}$ indicate energies measured for the barium covered plane. 
originating from the thin barium films. Probably the excited electrons are associated with the $\mathrm{Ba} 5 d$ states [2]. Finally, the difference between the two energies observed here $(-0.28 \mathrm{eV}$ and $-0.07 \mathrm{eV})$ amounts to about $0.2 \mathrm{eV}$, while suitable separation of peaks obtained by Plummer and Young [2] for single atoms on tungsten is about $0.3 \mathrm{eV}$.

\section{Acknowledgment}

This work was supported by Wroclaw University, No. 2016/W/IFD/92.

\section{References}

[1] L.D. Schmidt, J. Chem. Phys. 46, 3830 (1967).

[2] E.W. Plummer, R.D. Young, Phys. Rev. B 1, 2088 (1970).

[3] K. Jacobi, C. Astaldi, B. Frick, P. Geug, Phys. Rev. B 36, 3079 (1987).

[4] A. Lamouri, I.L. Krainsky, Surf. Sci. 278, 286 (1992).

[5] H. Neumann, Ch. Kleint, Ann. Phys. (Leipzig) 27, 273 (1971).

[6] T. Radon', Ch. Kleint, Surf. Sci. 60, 540 (1976).

[7] T. Radoń, S. Jaskólka, Surf. Sci. 231, 160 (1990).

[8] T. Radoń, S. Jaskółka, Surf. Sci. 247, 106 (1991).

[9] T. Radon', S. Jaskółka, Surf. Phys. 3, 166 (1988): Proc. 2nd Conf. Surf. Phys. Wroctaw (Poland) 1987, Eds. A. Gieroszyniski, J. Rutkowski, S. Sujak, Univ. of Eódź Publishing Co., Lódź 1988.

[10] Ch. Kleint, A. Modinos, Surf. Sci. 177, 389 (1986).

[11] N.E. Christensen, B. Feuerbacher, Phys. Rev. B 10, 2349 (1974).

[12] T. Radoń, S. Jaskółka, Surf. Sci. 200, 199 (1988). 